\title{
Towards optical fibre based Raman spectroscopy for the detection of surgical site infection
}

\author{
Alex J. Thompson ${ }^{1}$, Lukasz Koziej ${ }^{2}$, Huw D. Williams ${ }^{2}$, Daniel S. Elson ${ }^{1}$, Guang-Zhong Yang ${ }^{1}$ \\ ${ }^{1}$ Hamlyn Centre for Robotic Surgery, Imperial College London, South Kensington, London, SW7 2AZ, UK \\ ${ }^{2}$ Department of Life Sciences, Imperial College London, South Kensington, London, SW7 2AZ, UK
}

*Corresponding author: alex.thompson08@imperial.ac.uk

\begin{abstract}
Surgical site infections (SSIs) are common post-surgical complications that remain significant clinical problems, as they are associated with substantial mortality and morbidity. As such, there is significant interest in the development of minimally invasive techniques that permit early detection of SSIs. To this end, we are applying a compact, clinically deployable Raman spectrometer coupled to an optical fibre probe to the study of bacteria, with the long term goal of using Raman spectroscopy to detect infection in vivo. Our system comprises a $785 \mathrm{~nm}$ laser diode for excitation and a commercial (Ocean Optics, Inc.) Raman spectrometer for detection. Here we discuss the design, optimisation and validation of this system, and describe our first experiences interrogating bacterial cells (Escherichia coli) in vitro.
\end{abstract}

Key words: Raman scattering, SERS, spectroscopy, surgical site infection, bacteria, Escherichia coli.

\section{INTRODUCTION}

Surgical site infections (SSIs) - post-operative infections occurring in or near surgical sites within 30 days of the initial procedure - represent a significant clinical problem. In Europe, incidence rates can be as high as $20 \%$ depending on the surgical procedure [1] and SSIs remain a major cause of both morbidity and mortality [1-3]. Importantly, the transition from the original bacterial contamination to an established infection occurs early. However, SSIs are often detected much later (typically when the patient develops symptoms), meaning that further surgery can be required. This leads to increases in both the lengths of patients' hospital stays and the cost of treatment. Early detection of SSIs would permit more efficient treatment using antibiotics and would reduce the need for additional surgical interventions, thereby improving the experience of the patient and reducing the levels of both cost and mortality. As such, there is increasing interest in the development of minimally invasive techniques that can provide early diagnosis of SSIs, allowing effective antibiotic treatments to be administered. To this end, we are applying a compact, optical fibre based Raman spectrometer - that can be deployed in a clinical setting - to the study and detection of bacteria in vitro, with the aim of determining the potential of Raman spectroscopy for the early detection of SSI.

Raman spectroscopy and surface enhanced Raman spectroscopy (SERS) have been widely used in biomedical studies. In particular, Raman spectroscopy has been used for the detection and discrimination of bacteria, with applications in both hygiene and medicine. As early as 1980, Howard et al. reported the detection and identification of multiple bacteria in water using resonance Raman spectroscopy [4]. Since then many articles have been published discussing the use of Raman spectroscopy and/or SERS to detect bacteria and to discriminate between different species for a variety of applications (e.g. [5-9]). The use of optical fibres to deliver excitation and to collect the scattered Raman signal provides a route to minimally invasive medical use and Yang et al. recently reported the detection of the bacteria Shewanella oneidensis MR-1 in aqueous solution using a fibre-optic SERS probe [10]. Additionally, de Siqueira e Oliveira et al. [11] used an optical fibre based Raman probe to study multiple bacterial species that are involved in urinary tract infections (UTIs), a common type of SSI. Using this system, they were able to successfully discriminate between different pathogenic species using a diagnostic algorithm based on principal component analysis (PCA) of the acquired Raman spectra. Further investigation of the use of Raman spectroscopy in the study and diagnosis of UTIs has been performed by the research group of Costas Pitris [12-15]. In their research, the Pitris group used Raman spectroscopy to detect the presence of bacteria, to discriminate multiple bacterial species using PCA, and, finally, to 
assess the bacteria's response to antibiotic treatment [12-15]. Overall, this implies that Raman spectroscopy has the potential to become a useful clinical tool for early diagnosis of infection as it can permit both the detection and identification of relevant bacteria.

In this article we report the use of a compact, clinically deployable, bench-top Raman spectrometer coupled to a bifurcated optical fibre probe for the study of bacteria in vitro. We discuss the design and validation of this system and present preliminary in vitro data from Escherichia coli. Further work will involve the study of additional bacteria and the application of our spectrometer to clinical studies investigating the potential for point-of-care diagnosis of SSI.

\section{EXPERIMENTAL PROCEDURES}

\subsection{A compact optical fibre based Raman spectrometer}

Our compact, clinically deployable Raman spectrometer was configured as shown in figure 1(a). The emission from a continuous wave (CW) $785 \mathrm{~nm}$ laser diode (L785P090, Thorlabs Ltd., UK) - which was housed in a temperature controlled mount (LDM9T/M, Thorlabs Ltd., UK) - was coupled into the excitation channel of a bifurcated optical fibre probe (InPhotonics RIP-RPB-785-FC-SMA, Ocean Optics Inc., The Netherlands). This probe contained two multimode fibres: one $105 \mu \mathrm{m}$ diameter fibre for light delivery and one $200 \mu \mathrm{m}$ diameter fibre for light collection. At the distal end of the probe (which was common to both fibres), the excitation light exited the fibre and passed through a collimation lens. A bandpass filter and a dichroic filter were then used to remove any background fluorescence or Raman signal caused by the optical fibre itself. Finally, a second lens brought the light to a focus at a distance of $7.5 \mathrm{~mm}$ from the tip of the probe. The scattered Raman signal was then collected by the second lens and directed toward the collection fibre by the dichroic beam splitter. A mirror directed the light through a long pass filter assembly (which blocked any residual elastically scattered excitation light) and a third lens coupled the light into the $200 \mu \mathrm{m}$ collection fibre [16, 17]. The collection branch of the proximal end of the probe then delivered the light to a commercial Raman spectrometer (QEPro, Ocean Optics Inc., The Netherlands) for spectral detection.

(a)

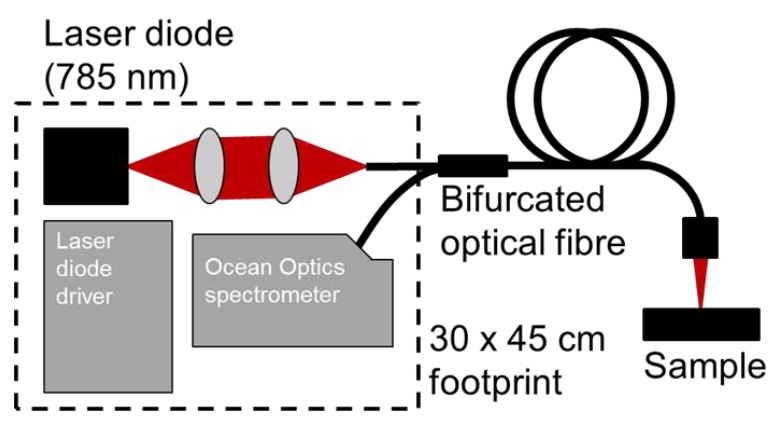

(b)

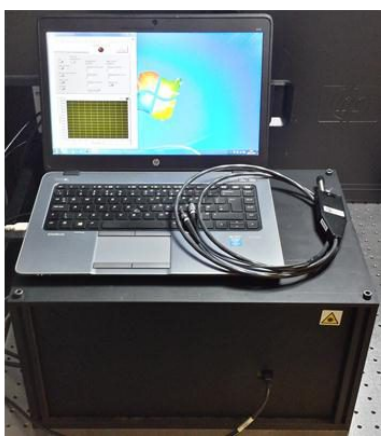

Figure 1. Schematic diagram (a) and photograph (b) of the compact, optical fibre based Raman spectrometer used in this study.

All optical components were mounted on a $30 \times 45 \mathrm{~cm}$ breadboard along with the driver for the laser diode (LDC 202 C, Thorlabs Ltd., UK) and a USB data acquisition box (NI USB-6009, National Instruments, USA) that allowed computer control of the on/off state of the laser. The entire system was contained within a light-tight black box and the only point at which laser light emerged from the system was at the distal tip of the fibre probe. For all measurements, the distal end of the probe was clamped above the sample in a custom made mount and fixed such that the laser emission was directed downward, allowing laser-safe use. The laser output and the spectrometer were both controlled by a laptop computer running software written in-house using LabVIEW 2014 (National Instruments, USA). A photograph of the fully housed system is shown in figure 1(b), where the compact, transportable nature of the device is evident.

To provide an acceptable signal to noise ratio (SNR), commercial SERS substrates (RAM-SERS-AU, Ocean Optics Inc., The Netherlands) were used to mount samples. For a typical measurement, a $10 \mu 1$ liquid sample was placed 
on the active area of a SERS substrate and illuminated with approximately $35 \mathrm{~mW}$ of optical power. Spectra were collected with acquisition times ranging from $0.1 \mathrm{~s}$ to several minutes. First, spectra from a $0.5 \mathrm{mM}$ aqueous solution of Rhodamine $6 \mathrm{G}$ were recorded in order to validate the system (i.e. to confirm that we observed Raman peaks at the expected frequencies). Second, spectra from live E. coli were recorded at two concentrations (approximately $10^{8}$ cells $/ \mathrm{ml}$ and $10^{9}$ cells $/ \mathrm{ml}$ respectively) with a variety of acquisition times. These experiments served to confirm that the system provided sufficient sensitivity to detect and study the Raman signatures of bacteria.

\subsection{E. coli sample preparation}

E. coli (DH5a strain, laboratory stocks) was streaked onto Luria Broth (LB) agar (15 g/l agar, Sigma-Aldrich, USA) and grown overnight at $37^{\circ} \mathrm{C}$. A single colony was used to inoculate $5 \mathrm{ml}$ of LB medium $(5 \mathrm{~g} / 1$ yeast extract, Fisher Scientific, UK; $5 \mathrm{~g} / \mathrm{l} \mathrm{NaCl}$, Sigma-Aldrich, USA; $10 \mathrm{~g} / \mathrm{l}$ tryptone, Fisher Scientific, UK), which was shaken overnight $(220 \mathrm{rpm})$ at $37^{\circ} \mathrm{C} .1 .1 \mathrm{ml}$ of the starter growth solution was then diluted in a 1:51 ratio with a further $55 \mathrm{ml}$ of fresh LB medium (producing a total volume of $56.1 \mathrm{ml}$ ). This was then allowed to shake at $37^{\circ} \mathrm{C}$ at $220 \mathrm{rpm}$ until OD600 (the absorbance at $600 \mathrm{~nm}$ ) of 0.6 was reached, corresponding to a concentration of approximately $10^{8}$ cells $/ \mathrm{ml}$. To concentrate the sample further, $50 \mathrm{ml}$ of growth was centrifuged for 20 minutes at room temperature at $2500 \mathrm{xg}$ (J6, Beckman Coulter Inc., USA) and the cells were then resuspended in $5 \mathrm{ml}$ of LB medium to produce a sample with a concentration 10 times higher than that of the original (i.e. approximately $10^{9}$ cells $/ \mathrm{ml}$ ). A $5 \mathrm{ml}$ sample of the original growth was maintained at the initial concentration of $10^{8} \mathrm{cells} / \mathrm{ml}$.

\section{RESULTS AND DISCUSSION}

\subsection{Surface enhanced Raman spectroscopy of Rhodamine 6G}

To validate our system, we measured SERS spectra of Rhodamine $6 \mathrm{G}$ and compared them to those presented in literature. Figure 2 shows SERS spectra acquired from a $0.5 \mathrm{mM}$ solution of Rhodamine $6 \mathrm{G}$ with acquisition times ranging from 0.1-10 s. In all cases, the observed peaks agree well with those reported elsewhere in the literature (e.g. [18]), indicating that our spectrometer successfully detects Raman signals from Rhodamine 6 G at a concentration of $0.5 \mathrm{mM}$. Furthermore, all peaks are clear above the background with a good SNR even using the shortest acquisition time of $0.1 \mathrm{~s}$ (see figure 2(b)).
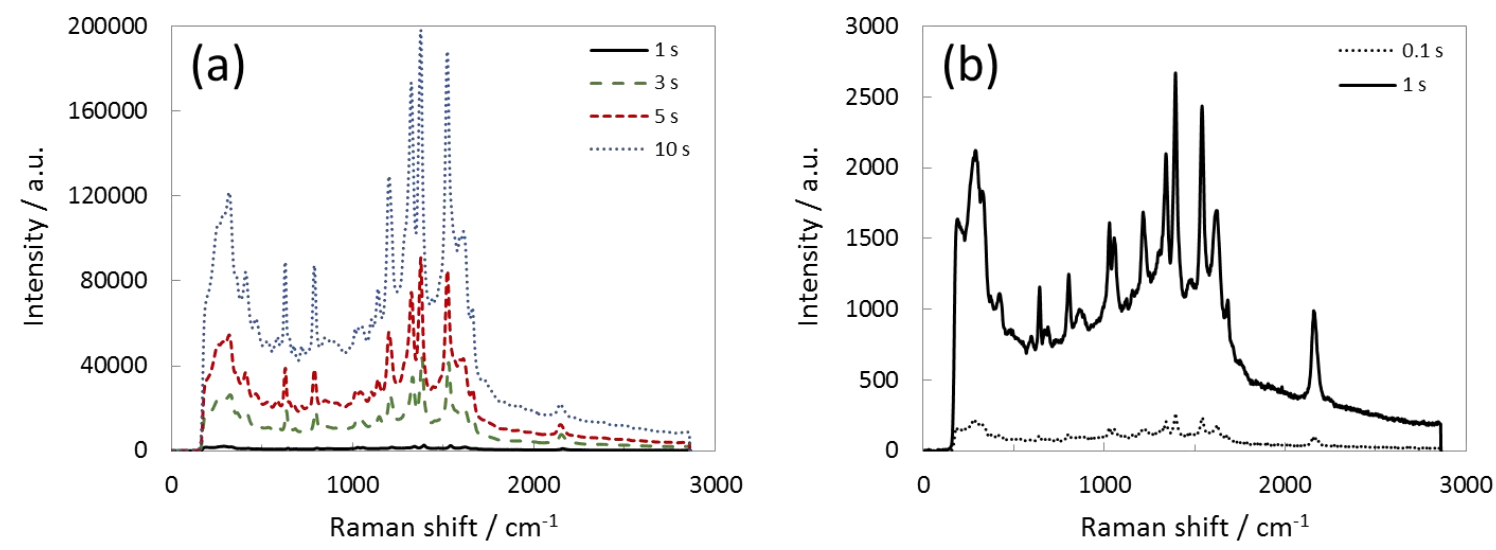

Figure 2. SERS spectra recorded in a $0.5 \mathrm{mM}$ sample of Rhodamine 6G. (a) Spectra recorded with acquisition times of 1, 3, 5 and $10 \mathrm{~s}$. (b) Spectra recorded with acquisition times of $0.1 \mathrm{~s}$ and $1 \mathrm{~s}$. The y-axis scale in (b) is smaller than that used in (a), revealing that the spectral peaks are clearly resolvable even using a $0.1 \mathrm{~s}$ integration time. Also note the clearer presence of background peaks in the spectra presented in (b), for example at approximately $1050 \mathrm{~cm}^{-1}$ and $2160 \mathrm{~cm}^{-1}$. These were attributed to the SERS substrate itself and were observed to decay over time. Hence, their relative intensities are weaker in the longer acquisitions shown in (a), which were recorded later. 


\subsection{Surface enhanced Raman spectroscopy of E. coli}

After validating our spectrometer using Rhodamine $6 \mathrm{G}$ we recorded a series of spectra from $E$. coli to assess the sensitivity of our system for the detection of bacteria. Figure 3 shows Raman spectra from E. coli recorded at two concentrations (approximately $10^{8}$ cells/ml and $10^{9}$ cells/ml) and using multiple acquisition times $(1 \mathrm{~s}, 10 \mathrm{~s}, 60 \mathrm{~s}$ and $300 \mathrm{~s}$ ). Background spectra recorded from a bare SERS substrate and the bacterial growth media are also presented. As shown in figure 3(a), while there are clear background signals from the SERS substrates and the growth media, spectral peaks from the E. coli are evident above this background. In particular, a series of 3-4 Raman peaks are clearly observed in the frequency range $1250-1500 \mathrm{~cm}^{-1}$ (indicated by the black arrows in figure 3). These peaks are just discernible at $10^{8}$ cells $/ \mathrm{ml}$ and are clearly detected at $10^{9}$ cells $/ \mathrm{ml}$. Additionally, the spectral locations are in qualitative agreement with previously published data (e.g. $[7,19,20])$, even though there is considerable variation within the literature. Importantly, the characteristic shape of the $E$. coli spectra can be resolved even at acquisition times as low as $1 \mathrm{~s}$. This is demonstrated in figures 3(b) and 3(c), which show spectra recorded from E. coli at a concentration of $10^{9} \mathrm{cells} / \mathrm{ml}$ with varying acquisition times. Using a $1 \mathrm{~s}$ integration time (figure 3(c)), the characteristic E. coli peaks in the $1250-1500 \mathrm{~cm}^{-1}$ frequency range are still clearly detected above the noise. This indicates the high sensitivity of our system, which will be vital when deploying this spectrometer (or a similar system) to clinical diagnostic studies of SSI where the concentration of bacteria will be considerably lower.
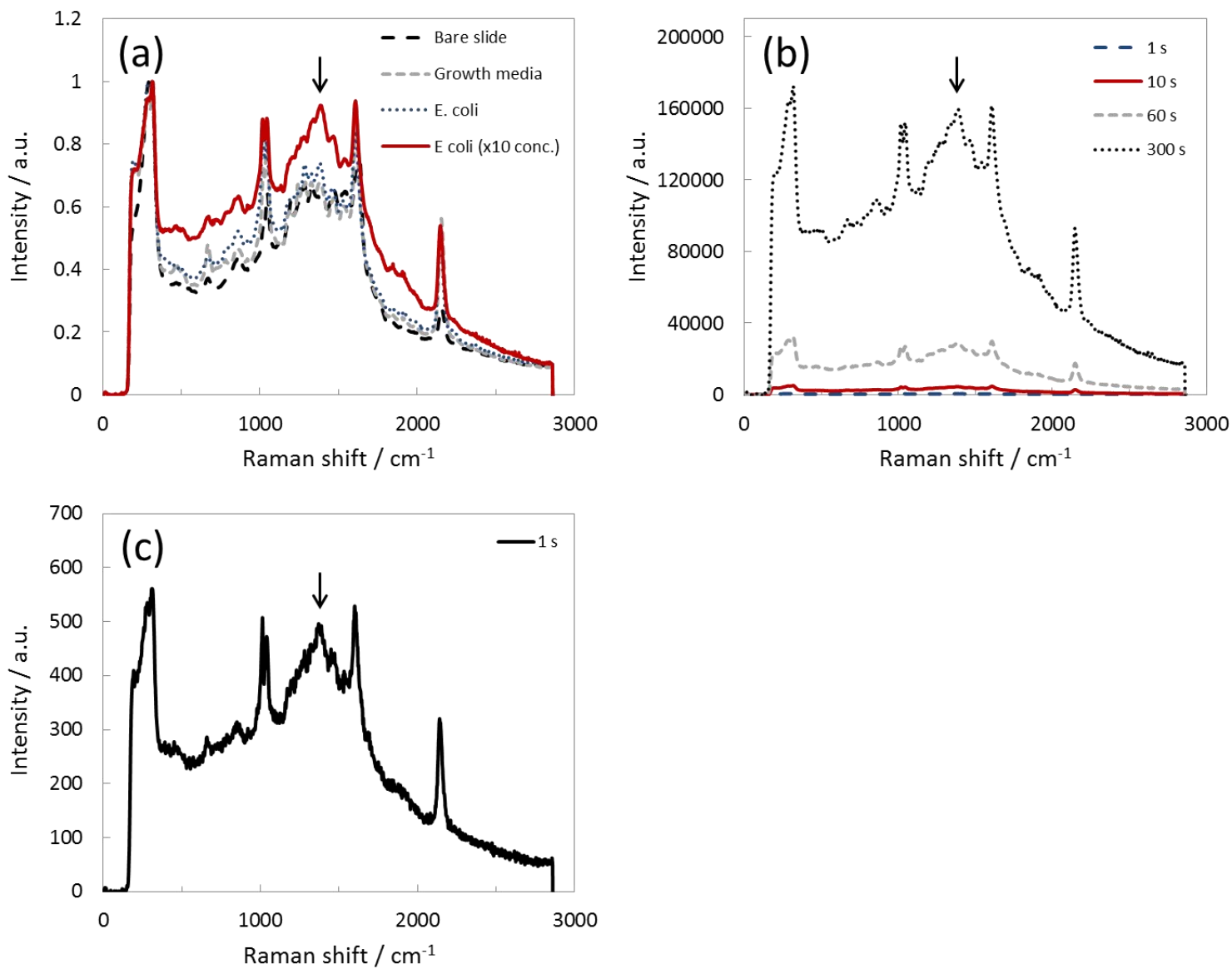

Figure 3. SERS spectra recorded in live E. coli. (a) Normalised spectra from a bare SERS substrate, blank bacterial growth media, and E. coli at concentrations of $10^{8}$ cells $/ \mathrm{ml}$ and $10^{9}$ cells $/ \mathrm{ml}$ (see key; $10^{9}$ cells $/ \mathrm{ml}$ concentration is labelled as ' $\mathrm{x} 10$ conc.'). Normalised spectra are shown in (a) to account for variations in intensity caused by fluctuations in laser power and differences in the spectral intensities observed when using different SERS substrates. (b) SERS spectra from E. coli at a concentration of $10^{9}$ cells $/ \mathrm{ml}$ with acquisition times of $1,10,60$ and $300 \mathrm{~s}$ respectively. (c) The $1 \mathrm{~s}$ acquisition shown in (b) presented on a smaller $y$-axis scale revealing that spectral peaks are clearly resolved even with this short integration time. The black arrows in (a-c) indicate the spectral peaks corresponding to E. coli. 
Overall, these results indicate that our compact, fibre-coupled Raman spectrometer provides sufficient sensitivity for the detection of $E$. coli in vitro. This is true when using acquisition times as low as $1 \mathrm{~s}$ and with a moderate laser excitation power of approximately $35 \mathrm{~mW}$. Further work will now involve the interrogation of a series of additional bacterial species in order to ascertain whether Raman spectroscopy has merit in the detection and identification of bacteria that are relevant to SSI.

\section{CONCLUSIONS}

We have presented preliminary data from an in vitro study of the Raman spectral signatures of live $E$. coli. This data was collected using a compact, fibre-optic Raman spectrometer that has been designed for clinical use. The use of optical fibres for light delivery and collection is important as it allows interrogation of remote samples, which is highly beneficial in medical applications. Additionally, Raman spectra (or SERS spectra) have rarely been recorded in bacterial samples using fibre-optic systems $[10,11]$ due to the reduced signal collection efficiencies and high background levels that such devices often suffer from. Here, using a fibre-optic spectrometer, we recorded Raman spectra from live E. coli and observed qualitative agreement with literature. Furthermore, we were able to discern the relevant spectral peaks over the background with acquisition times as low as $1 \mathrm{~s}$, indicating that our setup may be useful in the detection of bacteria at

low concentrations. Further work will now involve the investigation of additional bacterial species to assess the merit of fibre-optic Raman spectroscopy in the diagnosis of SSI.

\section{ACKNOWLEDGMENTS}

The authors gratefully acknowledge the funding for this work, which was provided by the Engineering and Physical Sciences Research Council grant EP/L014149/1.

\section{REFERENCES}

[1] Owens, C. D. and Stoessel, K., "Surgical site infections: Epidemiology, microbiology and prevention," Journal of Hospital Infection, 70, Supplement 2: p. 3-10 (2008)

[2] DiPiro, J. T., Martindale, R. G., Bakst, A., Vacani, P. F., Watson, P., and Miller, M. T., "Infection in surgical patients: Effects on mortality, hospitalization, and postdischarge care," American Journal of Health-System Pharmacy, 55(8): p. 777-781 (1998)

[3] Elgohari, S., Mihalkova, M., Wloch, C., Saei, A., Harris, R., Charlett, A., and Lamagni, T., "Surveillance of surgical site infections in NHS hospitals in england 2013/14", London: Public Health England, 2014, Available from: www.gov.uk/phe

[4] Howard, W. F., Nelson, W. H., and Sperry, J. F., "Resonance Raman method for the rapid detection and identification of bacteria in water," Applied Spectroscopy, 34(1): p. $72-75$ (1980)

[5] Laucks, M. L., Sengupta, A., Junge, K., Davis, E. J., and Swanson, B. D., "Comparison of psychro-active arctic marine bacteria and common mesophillic bacteria using surface-enhanced Raman spectroscopy," Applied Spectroscopy, 59(10): p. 1222-8 (2005)

[6] Sengupta, A., Laucks, M. L., and Davis, E. J., "Surface-enhanced Raman spectroscopy of bacteria and pollen," Applied Spectroscopy, 59(8): p. 1016-23 (2005)

[7] Sengupta, A., Mujacic, M., and Davis, E. J., "Detection of bacteria by surface-enhanced Raman spectroscopy," Analytical and Bioanalytical Chemistry, 386(5): p. 1379-1386 (2006)

[8] Kahraman, M., Zamaleeva, A. I., Fakhrullin, R. F., and Culha, M., "Layer-by-layer coating of bacteria with noble metal nanoparticles for surface-enhanced Raman scattering," Analytical and Bioanalytical Chemistry, 395(8): p. 2559-67 (2009)

[9] Sivanesan, A., Witkowska, E., Adamkiewicz, W., Dziewit, L., Kaminska, A., and Waluk, J., "Nanostructured silver-gold bimetallic SERS substrates for selective identification of bacteria in human blood," Analyst, 139(5): p. 1037-1043 (2014)

[10] Yang, X., Gu, C., Qian, F., Li, Y., and Zhang, J. Z., "Highly sensitive detection of proteins and bacteria in aqueous solution using surface-enhanced Raman scattering and optical fibers," Analytical Chemistry, 83(15): p. 5888-5894 (2011) 
[11] de Siqueira e Oliveira, F. S., Giana, H. E., and Silveira, L., "Discrimination of selected species of pathogenic bacteria using near-infrared Raman spectroscopy and principal components analysis," Journal of Biomedical Optics, 17(10): p. 107004 (2012)

[12] Kastanos, E. K., Kyriakides, A., Hadjigeorgiou, K., and Pitris, C., "A novel method for urinary tract infection diagnosis and antibiogram using Raman spectroscopy," Journal of Raman Spectroscopy, 41(9): p. 958-963 (2010)

[13] Kastanos, E., Kyriakides, A., Hadjigeorgiou, K., and Pitris, C., "A novel method for bacterial UTI diagnosis using Raman spectroscopy," International Journal of Spectroscopy, 2012 (2012)

[14] Hadjigeorgiou, K., Kastanos, E., and Pitris, C., Urinary tract infection (UTI) multi-bacteria multi-antibiotic testing using surface enhanced Raman spectroscopy (SERS), in: Optical Diagnostics and Sensing XIII: Toward Point-of-Care Diagnostics, SPIE Photonics West, 2013, San Francisco, CA, SPIE

[15] Kastanos, E., Hadjigeorgiou, K., and Pitris, C., Rapid identification of bacterial resistance to ciprofloxacin using surface enhanced Raman spectroscopy, in: Optical Diagnostics and Sensing XIV: Toward Point-of-Care Diagnostics, SPIE Photonics West, 2014, San Francisco, CA, SPIE

[16] InPhotonics, "RPB fiber optic Raman probe user's manual," InPhotonics, Inc.

[17] Forney, R. W. and Kawai, N. T., Dual function safety and calibration accessory for Raman and other spectroscopic sampling, 2003, Google Patents

[18] Yang, X., Shi, C., Newhouse, R., Zhang, J. Z., and Gu, C., "Hollow-core photonic crystal fibers for surfaceenhanced Raman scattering probes," International Journal of Optics, 2011: 754610 (2011)

[19] Zeiri, L., Bronk, B. V., Shabtai, Y., Eichler, J., and Efrima, S., "Surface-enhanced Raman spectroscopy as a tool for probing specific biochemical components in bacteria," Applied Spectroscopy, 58(1): p. 33-40 (2004)

[20] Jarvis, R. M., Brooker, A., and Goodacre, R., "Surface-enhanced Raman spectroscopy for bacterial discrimination utilizing a scanning electron microscope with a Raman spectroscopy interface," Analytical Chemistry, 76(17): p. 5198-5202 (2004) 